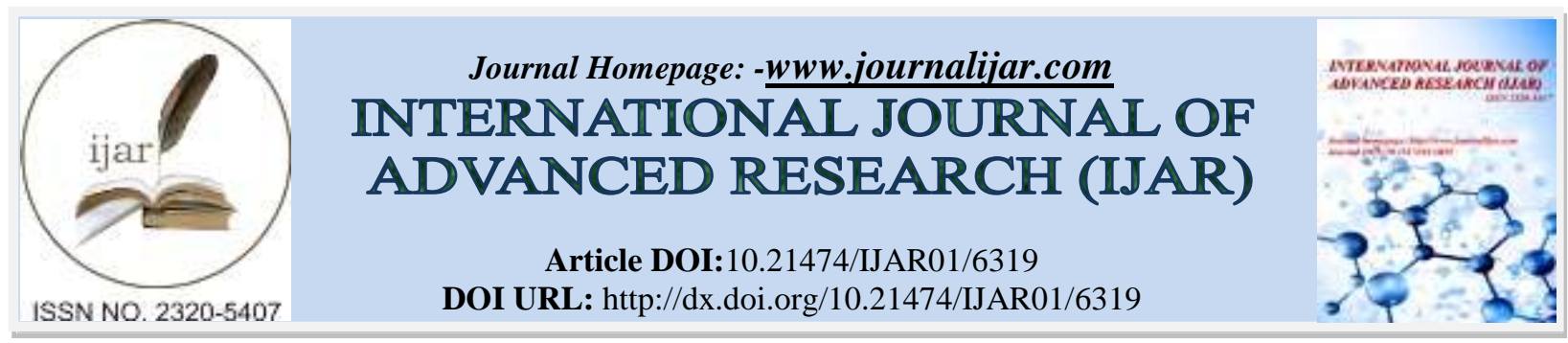

RESEARCH ARTICLE

\title{
BACTERIOLOGICAL AND MOLECULAR STUDIES ON BACTERIATRANSMITTED FROM FISHES TO HUMAN.
}

Ashraf A. Abd El-Tawaab'FatmaI. El-Hofy ${ }^{1}$ Adel M. El-Gamal $^{2}$ and Heba O. Ibrahim ${ }^{3}$.

1. Bacteriology, Immunology and Mycology Department Faculty of Veterinary Medicine, Benha University, Egypt.

2. Bacteriology Dept, Animal Health Research Institute, Kafr El-Sheikh branch. Egypt.

3. Veterinary Medicine Directorate, Kafr El-Sheikh branch. Egypt.

\section{Manuscript Info}

Manuscript History

Received: 15 November 2017

Final Accepted: 17 December 2017

Key words:-

Bacteria, molecular, transmission, fish and human.
(..........................

Published: January 2018

\begin{abstract}
A total of 1500 samples from 300 apparently healthy fish (280 Tilapia nilotica and 20 Catfish) were collected randomly from some markets and farms at Kafr El-sheikh governorate, Egypt. Also 50 human skin swabs were collected. These samples were collected to isolate and characterize bacteria transmitted from fish to human. In this study the prevalence of Staphylococcus aureus, Escherichia coli, Salmonella spp., Pseudomonas spp., Aeromonas spp. and Edwardseillatarda in Tilapia were $8.2,21.4,19,11.4,10$ and $1.1 \%$ respectively, the prevalence of theses pathogens in Catfish were 5, 75, 60, 15, 10 and $0 \%$ respectively while its prevalence in human were 8, 24, 20, 12, 0 and 0\% respectively. Escherichia coli serotype isolates from fish were O153, O1, O125 and O78. Salmonella serotype isolates from fish were Salmonellainganda, Salmonellatyphimurium and Salmonella Kentucky and Salmonellamolade. Results showed that all the eight isolates of Staphylococcusaureus subjected for determination of clfA gene were positive and negative to Hla gene, all the five Salmonella isolates were positive to invA and sefA genes and only one from four isolates of Escherichia coli was positive to eaeA gene and the four isolates were negative to Hly gene.
\end{abstract}

Copy Right, IJAR, 2018,. All rights reserved.

\section{Introduction:-}

Fish diseases are the major problems in fish farm industry. Disease cause economic losses because of high costs of treatment, fish mortality and zoonotic diseases occurred during handling the affected fish (Magdyet al., 2014).

Fish contain a lot of proteins, vitamins and unsaturated fatty acids. Fish considered the cheapest source of animal proteins.Staphylococcus species are the most important food borne opportunistic bacteria which isolated from fish samples, some of Staphylococcus species are potential pathogens, and high population of these bacteria indicates the degree of spoilage which might have undergoneand the general quality of fish (Ali 2014).

Escherichia coli in fish are considered as an indicator of sewage pollution. Most of the E.coli is normal inhabitants in the small intestine and they are non-pathogenic, meaning they do not cause disease in the intestine. E.coli spread

Corresponding Author:-Ashraf A. Abd El-Tawaab.

Address:-Bacteriology, Immunology and Mycology Department Faculty of Veterinary Medicine, 
outside the intestine cause disease. The pathogenic strains of E.coli may cause diarrhea by producing and releasing toxins and cause deaths in fish (Solimanet al., 2010).

Salmonella spp. isolated from freshwater fish such as Catfish. This bacterium has a great risk on human health. Salmonella spp. infections can be life-threatening, especially for the very young, the elderly, and for people with immune system problems(Budiatiet al., 2011).

Pseudomonas spp. are widely spread in natural sources of water and associated with septicaemia in aquatic animals. These bacteria are opportunistic pathogens, causing disease when the host exposed to stress (Magdyet al., 2014).

Aeromonasspp. is an opportunistic and zoonotic important bacterium. This bacteria cause diseases in both warm and cold blooded animals as a result of their virulence and pathogenicity. In human cause gastroenteritis, wound infections and chronic diarrhea( Kambleet al., 2012).

Edwardseillatarda is commonly classified as opportunistic, $E$. tarda is a serious pathogen of fish. This bacterium has a great zoonotic importance. Consumption of infected fish is a cause of gastroenteritis and meningitis (Lima et al., 2008).

Human infections caused by pathogens transmitted from fish or the aquatic environment are quite common depending on different reasons such as the season, patients' contact with fish, dietary habits and the immune system status of the exposed individual (Novotny et al., 2004).

With the increasing intensification of aquaculture production, diseases cause problems in the fish farming industry. Although vaccines are being developed and marketed, cannot be used as a universal disease control measure in aquaculture. During the last decades, antibiotics used for fish diseases management, improvement of growth and efficiency of feed conversion (Denevet al., 2009).

The aims of this study are to detect, isolate and characterize bacteria transmitted from fish to human.

\section{Materials and methods:-}

\section{Sampling:-}

Fish samples: (Eissaet al., 2010 and Hassan et al., 2012):-

A total of 1500 samples (300 skin swab, 300 gills samples, 300 liver samples, 300 spleen samples and 300 kidney samples) from 300 apparently healthy fish (280 Tilapia and 20 Cat fish) were collected randomly from some markets and farms in Kafr El-sheikh governorate (Egypt) during the period from January 2016 to May 2016. 140 Tilapia fish were collected from markets, 140 from farms and 20 Catfish were collected from markets. Fish samples were placed in strong, clean and aseptic bags then packed in column and surrounded with ice bags and brought to laboratory on the day of collection.

Human samples: (El-olemyet al., 2014):-

A total of 50 human skin swabs were collected with $5 \mathrm{ml}$ saline to avoid dryness of samples.

Bacteriological examination:-

Isolation of bacteria:-

Isolation of Staphylococcus spp. from fish: (Muset al., 2014):-

Skin swab samples were cultured on to nutrient broth with $7 \% \mathrm{NaCl}$. The inoculated tubes were incubated at $37^{\circ} \mathrm{C}$ for $24 \mathrm{hrs}$.

Nutrient broth samples were streaked on to Baired Parker and Mannitol salt agar plates and incubated overnight at $37^{\circ} \mathrm{C}$.

Isolation of E.coli, Salmonella spp., Pseudomonas spp., Aeromonas spp. and Edwardseilla spp. from fish: (Solimanet al., 2010), (Hassan et al., 2012) and (Elissaet al., 2010, El-hady and Samy 2011 and Cagatay and Sen 2014 ):-

Apiece of liver, spleen, kidney and gills were cultured separately on to MacConkey broth, Tetrathiothianate broth, and Tryptic Soya broth for isolation of E.coli spp., Salmonella spp., Pseudomonas spp., Aeromonas spp., and Edwardseilla spp. Skin swabs were cultured onto Tryptic Soya broth for isolation of Pseudomonas spp. only. The 
inoculated tubes were incubated at incubator. The MacConkey broth samples were streaked on to Eosine Methylene Blue agar for isolation of E.coli, the Tetrathiothianates broth samples were streaked on to Salmonella Shigella agar for salmonella isolation and the Tryptic Soyas broth samples were streaked on to pseudomonas agar for isolation of Pseudomonas spp., RimellerShotts agar for isolation of Aeromonas spp. and Edwardseillamedia for isolation of Edwardseilla spp. (selective plating) and incubated overnight at $37^{\circ} \mathrm{C}$.

Isolation of Staphylococcus spp., E.coli spp., Salmonella spp., Pseudomonas spp., Aeromonasspp. and Edwardseilla spp. from human skin swab samples: (El-olemy et al., 2014):-

Biochemical identification:-

The pure colonies of isolates were identified biochemically according to (Koneman et al., 1983, Quinn et al., 2002).

Serological Identification of E.coli serovars according to (Koket al., 1996) and Salmonella serovars according to Kauffman (1974).

In-Vitro antibiotic sensitivity of bacteria according to Srivani (2001).

Polymerase Chain Reaction (PCR):-

Extraction of DNA:-

According to QIAamp DNA mini kit instructions

Preparation of PCR Master Mix:-

According to Emerald Amp GT PCR mastermix (Takara) Code No. RR310Akit

Cycling conditions of the primers during cPCR:-

Temperature and time conditions of the two primers during PCR according to specific authors and Emerald Amp GT PCR mastermix (Takara) kit:-

They have specific sequence and amplify a specific product as shown in Table (1).

Table 1:- Oligonucleotide primers sequencesSource:Metabion (Germany).

\begin{tabular}{|c|c|c|c|c|}
\hline Target MO & Gene & Sequence & $\begin{array}{l}\text { Amplified } \\
\text { product }\end{array}$ & Reference \\
\hline \multirow[t]{4}{*}{ Salmonella } & \multirow[t]{2}{*}{$\operatorname{InvA}$} & GTGAAATTATCGCCACGTTCGGGCAA & \multirow[t]{2}{*}{$284 \mathrm{bp}$} & \multirow[t]{2}{*}{ Oliveira et al., 2003} \\
\hline & & TCATCGCACCGTCAAAGGAACC & & \\
\hline & \multirow[t]{2}{*}{ sefA } & GCAGCGGTTACTATTGCAGC & \multirow[t]{2}{*}{$310 \mathrm{bp}$} & \multirow{2}{*}{$\begin{array}{l}\text { Akbarmehret al., } \\
2010\end{array}$} \\
\hline & & TGTGACAGGGACATTTAGCG & & \\
\hline \multirow[t]{4}{*}{ S. aureus } & \multirow[t]{2}{*}{ clfA } & GCAAAATCCAGCACAACAGGAAACGA & \multirow[t]{2}{*}{$638 \mathrm{bp}$} & \multirow[t]{2}{*}{ Mason et al., 2001} \\
\hline & & CTTGATCTCCAGCCATAATTGGTGG & & \\
\hline & \multirow[t]{2}{*}{$H l a$} & GAAGTCTGGTGAAAACCCTGA & \multirow[t]{2}{*}{704 bp } & \multirow[t]{2}{*}{ Feiet al., 2011} \\
\hline & & TGAATCCTGTCGCTAATGCC & & \\
\hline \multirow[t]{4}{*}{ E. coli } & \multirow[t]{2}{*}{ eaeA } & ATGCTTAGTGCTGGTTTAGG & \multirow[t]{2}{*}{$248 \mathrm{bp}$} & \multirow{2}{*}{$\begin{array}{l}\text { Bisi-Johnson et al., } \\
2011\end{array}$} \\
\hline & & GCCTTCATCATTTCGCTTTC & & \\
\hline & \multirow[t]{2}{*}{ Hly } & AACAAGGATAAGCACTGTTCTGGCT & \multirow[t]{2}{*}{$1177 \mathrm{bp}$} & \multirow[t]{2}{*}{ Pivaet al., 2003} \\
\hline & & ACCATATAAGCGGTCATTCCCGTCA & & \\
\hline
\end{tabular}

D- DNA Molecular weight marker.

The ladder was mixed gently by pipetting up and down. $6 \mu$ l of the required ladder were directly loaded.

E- Agarose gel electrophoreses (Sambrooket al., 1989).

\section{Results:-}

A total of 266 bacterial isolates from fish and 39 bacterial isolates from human were obtained from the examined samples.

E.coli was the predominant bacteria isolated from Tilapia with an incidence (21.4\%) followed by Staphylococcus spp. (19.6\%), Salmonella spp. (19\%), Pseudomonas spp. (11.4\%), Aeromonas spp. (10\%) and Edwardseillatarda $(1.1 \%)$ as shown in (Table 2). 
E.coli was the predominant bacteria isolated from Catfish (75\%) followed by Salmonella spp. (60\%), Staphylococcus spp. (15\%), Pseudomonas spp. (15\%), Aeromonas spp. (10\%) and there is no Edwardseilla spp. were isolated from Catfish as shown in (Table 3).

E.coli was the predominant bacteria were isolated from human (24\%), (20\%) of isolates were from sellers at markets and $(28 \%)$ from workers at farms. Followed by Staphylococcus spp. (22\%), (24\%) of isolates were from sellers at markets and (20\%) from workers at farms. Salmonella spp. (20\%). (16\%) of isolates from sellers at markets and (24\%) from workers at farms. Pseudomonas spp. (12\%). (16\%) of isolates from sellers at markets and (8\%) from workers at farms. No Aeromonas spp. and Edwardseilla spp. were isolated from human as shown in (Table 4).

Ten E.coli isolates were serotyped, six from Tilapia and four from human, serological identification revealed that five isolates from Tilapia were belonging to (O153, O1, O125 and 2 O78). Three isolates from human belonging to $(\mathrm{O} 153, \mathrm{O} 26$ and $\mathrm{O} 78)$ as shown in (Table 5).

Ten Salmonella isolates were serotyped, six from Tilapia fish and four from human. Serological identification revealed that four isolates from Tilapia belonging to $S$. inganda, $S$. typhimurium, $S$. kentucky and $S$. molade. Two isolates from human were belonging to $S$. typhimurium and $S$. enteritidis as shown in (Table 6).

E.coli O153, O1 isolates were resistant to Flumequine but E.coli O125 isolates were sensitive to Flumequine. E.coli O78, O26 isolates were resistant to Doxycillin but E.coliO78 from human was resistant to Ciprofloxacin and Chloramphenicol. It was shown that $S$. kentucky, $S$. molade and $S$. enteritidis were sensitive to Amoxicillin. But $S$. inganda and $S$. typhimurium were resistant to Penicillin (Table 7).

Identification of clfA and Hla virulence genes of eight Staphylococcus aureus isolates and the results revealed that all the isolates contain clfA gene but not contain Hla gene as shown in (Table 8) and (Figures 1,2).

Identification of eaeA and Hly virulence genes of four E.coli isolates that were serotyped and the results revealed that only one isolate contain eaeA gene as shown in (Table 9) and (Figures 3,4).

Identification of invA and sefA virulence genes of five Salmonella isolates that were serotyped and the results revealed that all the isolates contain both genes as shown in (Table 10) and (Figures 5, 6).

Table 2:- Incidence of bacteria isolated from Fish (280 Tilapia and 20 Catfish).

\begin{tabular}{|c|c|c|c|c|c|c|c|c|c|c|c|c|c|c|c|}
\hline \multirow{3}{*}{$\begin{array}{l}\text { Type } \\
\text { of fish }\end{array}$} & \multirow{3}{*}{$\begin{array}{l}\text { Number } \\
\text { Of } \\
\text { Examined } \\
\text { Fish }\end{array}$} & \multicolumn{14}{|c|}{ Bacteria } \\
\hline & & \multicolumn{2}{|c|}{ Staph spp. } & \multicolumn{2}{|c|}{ E.coli } & \multicolumn{2}{|c|}{ Salmonella } & \multicolumn{2}{|c|}{$\begin{array}{l}\text { Pseudomonas } \\
\text { spp. }\end{array}$} & \multicolumn{2}{|c|}{$\begin{array}{l}\text { Aeromonas } \\
\text { spp. }\end{array}$} & \multicolumn{2}{|c|}{$\begin{array}{l}\text { Edwardseilla } \\
\text { tarda }\end{array}$} & \multicolumn{2}{|c|}{ Total } \\
\hline & & +ve & $\%$ & $+\mathrm{ve}$ & $\%$ & $+\mathrm{ve}$ & $\%$ & +ve & $\%$ & $+\mathrm{ve}$ & $\%$ & $+\mathrm{ve}$ & $\%$ & $+\mathrm{ve}$ & $\%$ \\
\hline $\begin{array}{l}\text { Tilapia } \\
\& \\
\text { Catfish } \\
\end{array}$ & 300 & 58 & 19.3 & 75 & 25 & 65 & 21.7 & 35 & 11.7 & 30 & 10 & 3 & 1 & 266 & 88.7 \\
\hline Tilapia & 280 & 55 & 19.6 & 60 & 21.4 & 53 & 19 & 32 & 11.4 & 28 & 10 & 3 & 1.1 & 231 & 82.5 \\
\hline $\begin{array}{l}\text { Tilapia } \\
\text { from } \\
\text { farms }\end{array}$ & 140 & 23 & 16.4 & 35 & 25 & 23 & 16.4 & 17 & 12.1 & 13 & 9.2 & 0 & 0 & 111 & 79.3 \\
\hline
\end{tabular}

Table 3:- Incidence of bacteria isolated from Tilapia and Catfish collected from markets.

\begin{tabular}{|c|c|c|c|c|c|c|c|c|c|c|c|c|c|c|c|}
\hline \multirow{3}{*}{$\begin{array}{l}\text { Type } \\
\text { of fish }\end{array}$} & \multirow{3}{*}{$\begin{array}{l}\text { Number } \\
\text { Of } \\
\text { Examined } \\
\text { Fish }\end{array}$} & \multicolumn{14}{|c|}{ Bacteria } \\
\hline & & \multicolumn{2}{|c|}{ Staph. spp. } & \multicolumn{2}{|c|}{ E.coli } & \multicolumn{2}{|c|}{ Salmonella } & \multicolumn{2}{|c|}{$\begin{array}{l}\text { Pseudomonas } \\
\text { spp. }\end{array}$} & \multicolumn{2}{|c|}{$\begin{array}{l}\text { Aeromonas } \\
\text { spp. }\end{array}$} & \multicolumn{2}{|c|}{$\begin{array}{l}\text { Edwardseilla } \\
\text { tarda }\end{array}$} & \multicolumn{2}{|c|}{ Total } \\
\hline & & $+\mathrm{ve}$ & $\%$ & $+\mathrm{ve}$ & $\%$ & $+\mathrm{ve}$ & $\%$ & $+\mathrm{ve}$ & $\%$ & $+\mathrm{ve}$ & $\%$ & $+\mathrm{ve}$ & $\%$ & $+\mathrm{ve}$ & $\%$ \\
\hline Tilapia & 140 & 32 & 22.8 & 25 & 17.8 & 30 & 21.4 & 15 & 10.7 & 15 & 10.7 & 3 & 2.1 & 120 & 85.7 \\
\hline Catfish & 20 & 3 & 15 & 15 & 75 & 12 & 60 & 3 & 15 & 2 & 10 & 0 & 0 & 35 & 175 \\
\hline
\end{tabular}

Table 4:- Incidence of bacteria isolated from human.

\begin{tabular}{|c|c|c|c|c|c|c|c|c|}
\hline \multirow{2}{*}{$\begin{array}{l}\text { Sellers } \\
\text { and } \\
\text { workers }\end{array}$} & \multirow{2}{*}{$\begin{array}{l}\text { Number } \\
\text { of } \\
\text { examined }\end{array}$} & \multicolumn{6}{|c|}{ Isolation rate or incidence of bacteria isolated from human } & \multirow{2}{*}{$\begin{array}{l}\text { Total Number } \\
\text { and \% Per } \\
\text { Fish }\end{array}$} \\
\hline & & Staph spp. & E.coli & Salmonella & $\begin{array}{l}\text { Pseudomonas } \\
\text { spp. }\end{array}$ & $\begin{array}{l}\text { Aeromonas } \\
\text { spp. }\end{array}$ & $\begin{array}{l}\text { Edwardseilla } \\
\text { tarda }\end{array}$ & \\
\hline
\end{tabular}




\begin{tabular}{|l|l|l|l|l|l|l|l|l|l|l|l|l|l|l|l|}
\hline & sample & ve + & $\%$ & ve + & $\%$ & ve + & $\%$ & ve + & $\%$ & ve + & $\%$ & ve + & $\%$ & ve + & $\%$ \\
\hline $\begin{array}{l}\text { From } \\
\text { markets }\end{array}$ & 25 & 6 & 24 & 5 & 20 & 4 & 16 & 4 & 16 & 0 & 0 & 0 & 0 & 19 & 76 \\
\hline $\begin{array}{l}\text { From } \\
\text { farms }\end{array}$ & 25 & 5 & 20 & 7 & 28 & 6 & 24 & 2 & 8 & 0 & 0 & 0 & 0 & 20 & 80 \\
\hline Total & 50 & 11 & 22 & 12 & 24 & 10 & 20 & 6 & 12 & 0 & 0 & 0 & 0 & 39 & 78 \\
\hline
\end{tabular}

Table5:-Results of serological identification of E.coli isolates.

\begin{tabular}{|l|l|l|l|}
\hline Serial No. & Identified Bacterium & Serodiagnosis & Strain characterization \\
\hline 1 & E.coli(F) & $\mathrm{O} 153: \mathrm{H} 2$ & EPEC \\
\hline 2 & E.coli(F) & $\mathrm{O} 1: \mathrm{H} 7$ & EPEC \\
\hline 3 & E.coli(F) & $\mathrm{O} 125: \mathrm{H} 21$ & ETEC \\
\hline 4 & E.coli $(\mathrm{F})$ & $\mathrm{O} 78$ & EPEC \\
\hline 5 & E.coli(F) & $\mathrm{O} 78$ & EPEC \\
\hline 6 & E.coli $(\mathrm{H})$ & $\mathrm{O} 153: \mathrm{H} 2$ & EPEC \\
\hline 7 & E.coli(H) & $\mathrm{O} 26: \mathrm{H} 11$ & EHEC \\
\hline 8 & E.coli $(\mathrm{H})$ & $\mathrm{O} 78$ & EPEC \\
\hline 9,10 not $E \cdot \operatorname{coli}$ & & & \\
\hline
\end{tabular}

(F) E.coli isolated from fish.

(H) E.coli isolated from human.

Table 6:- Results of serological identification of Salmonella enterica isolates.

\begin{tabular}{|l|l|l|l|l|}
\hline Serial No. & Identified strains & \multirow{2}{*}{ Group } & \multicolumn{3}{l|}{ Antigenic structure } \\
\cline { 4 - 5 } & & & O & H \\
\hline 1 & Salmonella inganda(F) & C1 & 6,7 & $\mathrm{Z} 10: 1,5$ \\
\hline 2 & Salmonella typhimurium(F) & B & $1,4,5,12$ & $\mathrm{i}: 1,2$ \\
\hline 3 & Salmonella kentucky(F) & $\mathrm{C} 3$ & 8,20 & $\mathrm{i}: \mathrm{Z6}$ \\
\hline 4 & Salmonella molade(F) & $\mathrm{C} 2$ & 8,20 & $\mathrm{Z} 10: \mathrm{Z} 6$ \\
\hline 6 & Salmonella typhimurium(H) $(\mathrm{H})$ & $\mathrm{B}$ & $1,4,5,12$ & $\mathrm{i}: 1,2$ \\
\hline $7,8,9,10$ not Salmonella & D1 & $1,9,12$ & $\mathrm{~g}, \mathrm{~m}:-$ \\
\hline
\end{tabular}

(F) Salmonella isolated from fish.

(H) Salmonella isolated from human.

Table 7:- Results of sensitivity test of E.coli and Salmonella isolates.

\begin{tabular}{|c|c|c|c|c|c|c|c|c|c|c|c|c|c|c|c|}
\hline $\begin{array}{l}\text { Antimicrobial } \\
\text { agents }\end{array}$ & $\begin{array}{l}\text { Diffusion } \\
\text { zone } \\
\text { break } \\
\text { point } \\
(\mathrm{mm})\end{array}$ & 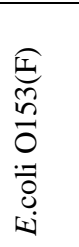 & 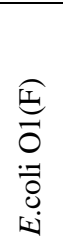 & 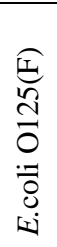 & 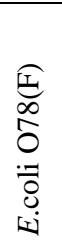 & 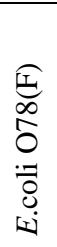 & 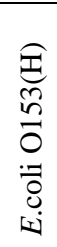 & 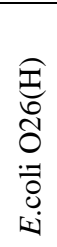 & 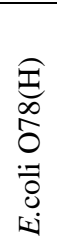 & 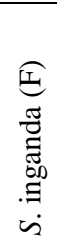 & 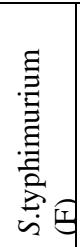 & 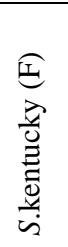 & $\begin{array}{l}\text { If } \\
\frac{0}{0} \\
\frac{\pi}{0} \\
\Xi \\
\dot{b}\end{array}$ & 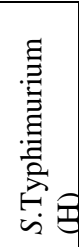 & 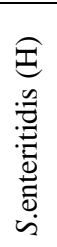 \\
\hline Amoxicillin(AML) & $14 \leq$ & $20(\mathrm{~S})$ & $\begin{array}{l}19( \\
S)\end{array}$ & $\begin{array}{l}21( \\
S)\end{array}$ & $\begin{array}{l}19( \\
\text { S) }\end{array}$ & $\begin{array}{l}19( \\
S)\end{array}$ & $\begin{array}{l}21( \\
S)\end{array}$ & $\begin{array}{l}19( \\
\text { S) }\end{array}$ & $\begin{array}{l}22( \\
S)\end{array}$ & $\begin{array}{l}21( \\
S)\end{array}$ & )$^{16(I}$ & $\begin{array}{l}22( \\
S)\end{array}$ & $\begin{array}{l}22( \\
S)\end{array}$ & $\begin{array}{l}21( \\
S)\end{array}$ & $\begin{array}{l}21( \\
S)\end{array}$ \\
\hline Penicillin $(\mathrm{P})$ & $20 \leq$ & $29(\mathrm{~S})$ & $\begin{array}{l}31( \\
\mathrm{S})\end{array}$ & $\begin{array}{l}30( \\
\mathrm{S})\end{array}$ & $\begin{array}{l}21(\mathrm{I} \\
{ }^{2}\end{array}$ & $\begin{array}{l}23( \\
\mathrm{I})\end{array}$ & $\begin{array}{l}28( \\
\mathrm{S})\end{array}$ & $\begin{array}{l}33( \\
\mathrm{S}) \\
\end{array}$ & $\begin{array}{l}30( \\
\mathrm{S})\end{array}$ & $\begin{array}{l}8(\mathrm{R} \\
) \\
\end{array}$ & $\begin{array}{l}9(\mathrm{R} \\
) \\
\end{array}$ & $\begin{array}{l}30( \\
\mathrm{S})\end{array}$ & $\begin{array}{l}22(\mathrm{I} \\
)\end{array}$ & $\begin{array}{l}33( \\
\mathrm{S}) \\
\end{array}$ & $\begin{array}{l}31( \\
\text { S) }\end{array}$ \\
\hline Ciprofloxacin(CP) & $12 \leq$ & $17(\mathrm{~S})$ & $\begin{array}{l}5(\mathrm{R} \\
)\end{array}$ & $\begin{array}{l}8(\mathrm{R} \\
)\end{array}$ & )$^{15(\mathrm{I}}$ & $\begin{array}{l}19( \\
\text { S) }\end{array}$ & $\begin{array}{l}16( \\
\text { I) }\end{array}$ & $\begin{array}{l}19( \\
\text { S) }\end{array}$ & $\begin{array}{l}11( \\
R)\end{array}$ & $\begin{array}{l}18( \\
\text { I) }\end{array}$ & )$^{14(\mathrm{I}}$ & $\begin{array}{l}15(\mathrm{I} \\
{ }^{2}\end{array}$ & $\begin{array}{l}10( \\
R)\end{array}$ & $\begin{array}{l}10( \\
R)\end{array}$ & $\begin{array}{l}10( \\
R)\end{array}$ \\
\hline Chloramphenicol(C) & $15 \leq$ & $18(\mathrm{~S})$ & $\begin{array}{l}10( \\
\mathrm{R})\end{array}$ & $\begin{array}{l}8(\mathrm{R} \\
)\end{array}$ & )$^{16(I}$ & $\begin{array}{l}17( \\
\mathrm{I})\end{array}$ & $\begin{array}{l}15( \\
\mathrm{I})\end{array}$ & $\begin{array}{l}\text { 15( } \\
\text { I) }\end{array}$ & $\begin{array}{l}4(\mathrm{R} \\
)^{2}\end{array}$ & $\begin{array}{l}20( \\
S)\end{array}$ & $\begin{array}{l}9(\mathrm{R} \\
{ }^{2}\end{array}$ & $\begin{array}{l}19( \\
\text { S) }\end{array}$ & $\begin{array}{l}21( \\
S)\end{array}$ & $\begin{array}{l}22( \\
S)\end{array}$ & $\begin{array}{l}20( \\
S)\end{array}$ \\
\hline Erythromycin(E) & $13 \leq$ & $23(\mathrm{~S})$ & $\begin{array}{l}24( \\
S)\end{array}$ & $\begin{array}{l}25( \\
S)\end{array}$ & $\begin{array}{l}11( \\
\mathrm{R})\end{array}$ & $\begin{array}{l}3(\mathrm{R} \\
{ }^{2}\end{array}$ & $\begin{array}{l}5(\mathrm{R} \\
)\end{array}$ & $\begin{array}{l}8(\mathrm{R} \\
)\end{array}$ & $\begin{array}{l}22( \\
S)\end{array}$ & $\begin{array}{l}8(\mathrm{R} \\
)\end{array}$ & $\begin{array}{l}10( \\
R)\end{array}$ & $\begin{array}{l}8(\mathrm{R} \\
)\end{array}$ & $\begin{array}{l}23( \\
S)\end{array}$ & $\begin{array}{l}23( \\
S)\end{array}$ & $\begin{array}{l}25( \\
S)\end{array}$ \\
\hline Neomycin(N) & $12 \leq$ & $19(\mathrm{~S})$ & $\begin{array}{l}18( \\
S)\end{array}$ & $\begin{array}{l}17( \\
S)\end{array}$ & )$^{13(I}$ & $\begin{array}{l}19( \\
S)\end{array}$ & $\begin{array}{l}18( \\
\text { S) }\end{array}$ & $\begin{array}{l}19( \\
\text { S) }\end{array}$ & $\begin{array}{l}19( \\
\text { S) }\end{array}$ & $\begin{array}{l}7(\mathrm{R} \\
{ }^{2}\end{array}$ & $\begin{array}{l}15(\mathrm{I} \\
{ }^{2}\end{array}$ & $\begin{array}{l}12( \\
R)\end{array}$ & $\begin{array}{l}22( \\
S)\end{array}$ & $\begin{array}{l}15(\mathrm{I} \\
{ }^{2}\end{array}$ & $\begin{array}{l}20( \\
S)\end{array}$ \\
\hline Doxacillin(DO) & $16 \leq$ & 17(I) & $\begin{array}{l}20( \\
\mathrm{S})\end{array}$ & $\begin{array}{l}17( \\
\mathrm{I})\end{array}$ & $\begin{array}{l}21( \\
\mathrm{S})\end{array}$ & $\begin{array}{l}20( \\
S)\end{array}$ & $\begin{array}{l}19( \\
\text { S) }\end{array}$ & $\begin{array}{l}17( \\
\mathrm{I})\end{array}$ & $\begin{array}{l}20( \\
S)\end{array}$ & $\begin{array}{l}19( \\
S)\end{array}$ & $\begin{array}{l}17(\mathrm{I} \\
\end{array}$ & $\begin{array}{l}20( \\
S)\end{array}$ & $\begin{array}{l}21( \\
S)\end{array}$ & $\begin{array}{l}19( \\
\text { S) }\end{array}$ & $\begin{array}{l}17(\mathrm{I} \\
{ }^{2}\end{array}$ \\
\hline Flumquine(UB) & $15 \leq$ & $18(\mathrm{~S})$ & $\begin{array}{l})^{13(\mathrm{I}} \\
\end{array}$ & $\begin{array}{l}20( \\
\text { S) }\end{array}$ & $\begin{array}{l}\text { 6(R } \\
)^{2}\end{array}$ & $\begin{array}{l}21( \\
S)\end{array}$ & $\begin{array}{l}22( \\
S)\end{array}$ & $\begin{array}{l}8(\mathrm{R} \\
)\end{array}$ & $\begin{array}{l}17( \\
S)\end{array}$ & $\begin{array}{l}13( \\
\mathrm{I})\end{array}$ & $\begin{array}{l}16( \\
\text { S) }\end{array}$ & $\begin{array}{l}13(\mathrm{I} \\
)^{2}\end{array}$ & $\begin{array}{l}18( \\
\mathrm{S})\end{array}$ & $\begin{array}{l}20( \\
\text { S) }\end{array}$ & $\begin{array}{l}13(\mathrm{I} \\
)^{13}\end{array}$ \\
\hline
\end{tabular}




\begin{tabular}{|l|l|l|l|l|l|l|l|l|l|l|l|l|l|l|l|}
\hline $\begin{array}{l}\text { Sulphamethoxazol+ } \\
\text { trimethoprime(SXT) }\end{array}$ & $10 \leq$ & $8(\mathrm{R})$ & $4(\mathrm{R}$ & 16( & 18( & 12( & 19( & 20( & 20( & 17( & 18( & 20( & 16( & 17( \\
$\mathrm{S}$ & & $\mathrm{S}$ & $\mathrm{S})$ & $\mathrm{S})$ & $\mathrm{I})$ & $\mathrm{S})$ & $\mathrm{S})$ & $\mathrm{S})$ & $\mathrm{S})$ & $\mathrm{S})$ & $\mathrm{S})$ & $\mathrm{S})$ & $\mathrm{S})$ & $\mathrm{S})$ \\
\hline
\end{tabular}

(F)Bacteriaisolated from fish

(H) Bacteriaisolated from human

Table 8:- Results of molecular identification of Hla and clfA gene of Staph.aureus.

\begin{tabular}{|l|l|l|l|}
\hline Target MO & Sample & Results & clfA \\
\cline { 2 - 4 } & & Hla & + \\
\hline \multirow{5}{*}{ S. aureus } & 1 & - & + \\
\cline { 2 - 4 } & 2 & - & + \\
\cline { 2 - 4 } & 3 & - & + \\
\cline { 2 - 4 } & 4 & - & + \\
\cline { 2 - 4 } & 5 & - & + \\
\cline { 2 - 4 } & 6 & - & + \\
\cline { 2 - 4 } & 7 & - & + \\
\hline
\end{tabular}

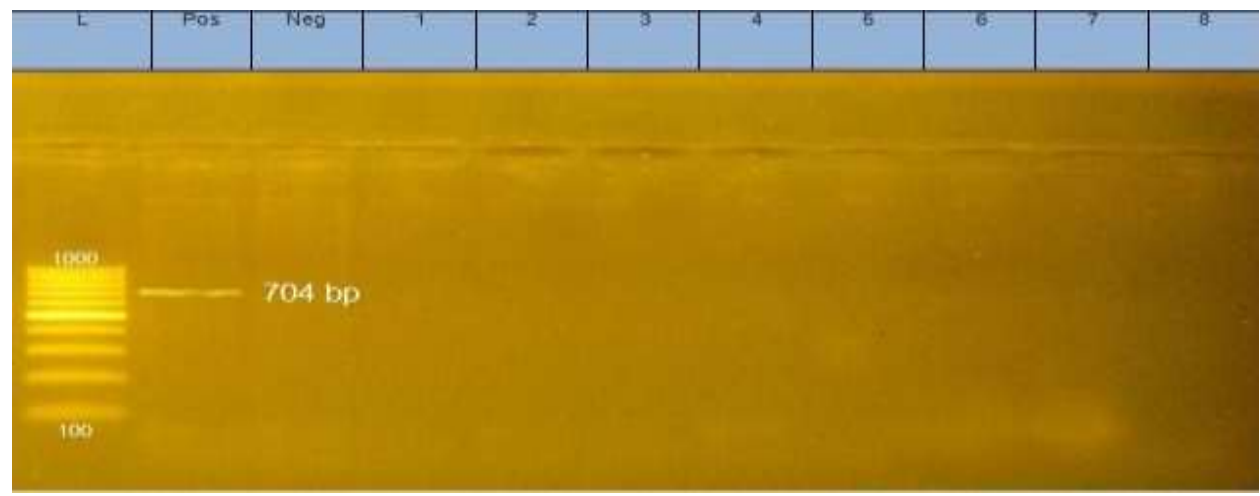

Fig 1:-Agrose gel electrophoresis of PCR amplified products of virulence gene. Lane L: DNA molecular size marker (100bP), lane Neg: Negative control, lane Pos: Positive control of Hla virulence gene of Staphylococcusaureus. The size in base pairs (704bP) of PCR product is indicated for the bands.

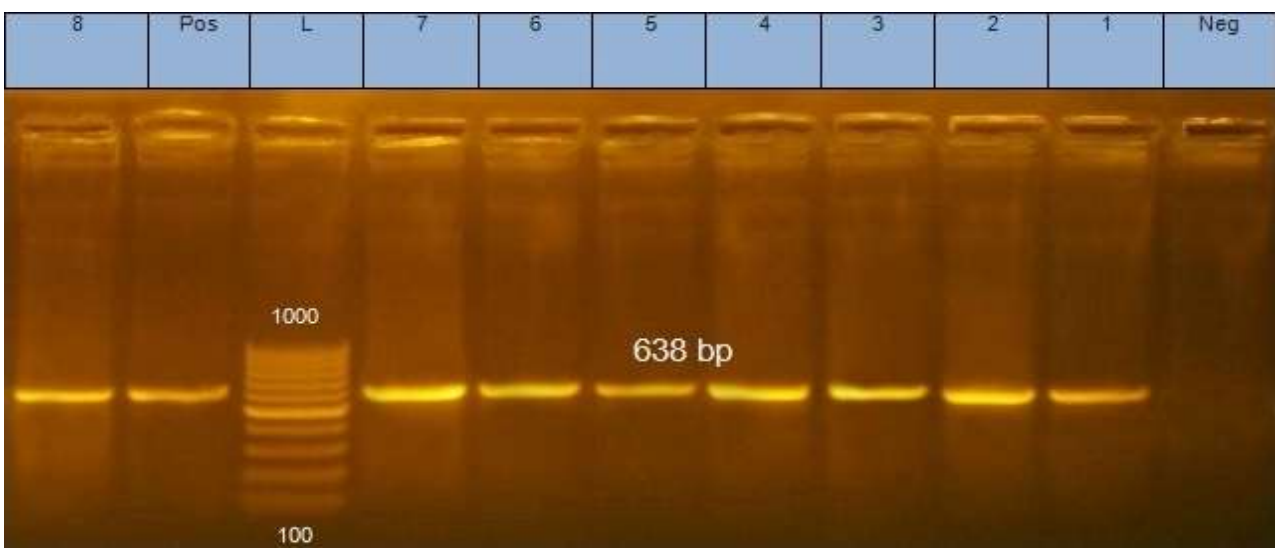

Fig 2:-Agrose gel electrophoresis of PCR amplified products of virulence gene. Lane L: DNA molecular size marker (100bP), lane Neg: Negative control, lane Pos: Positive control, lane 1, 2, 3, 4, 5, 6, 7, and 8: clfA virulence gene of Staphylococcusaureus. The size in base pairs $(683 \mathrm{bP})$ of PCR product is indicated for the bands.

Table 9:- Results of molecular identification of eaeA and Hlygene of E.coli.

\begin{tabular}{|l|l|l|l|}
\hline Target MO & Sample & Results & Hly \\
\cline { 3 - 4 } & & eaeA & - \\
\hline \multirow{2}{*}{ E. coli } & 1 & - & - \\
\cline { 2 - 4 } & 2 & + & \\
\hline
\end{tabular}




\begin{tabular}{|l|l|l|l|}
\hline \multirow{2}{*}{} & 3 & - & - \\
\cline { 2 - 4 } & 4 & - & - \\
\hline
\end{tabular}

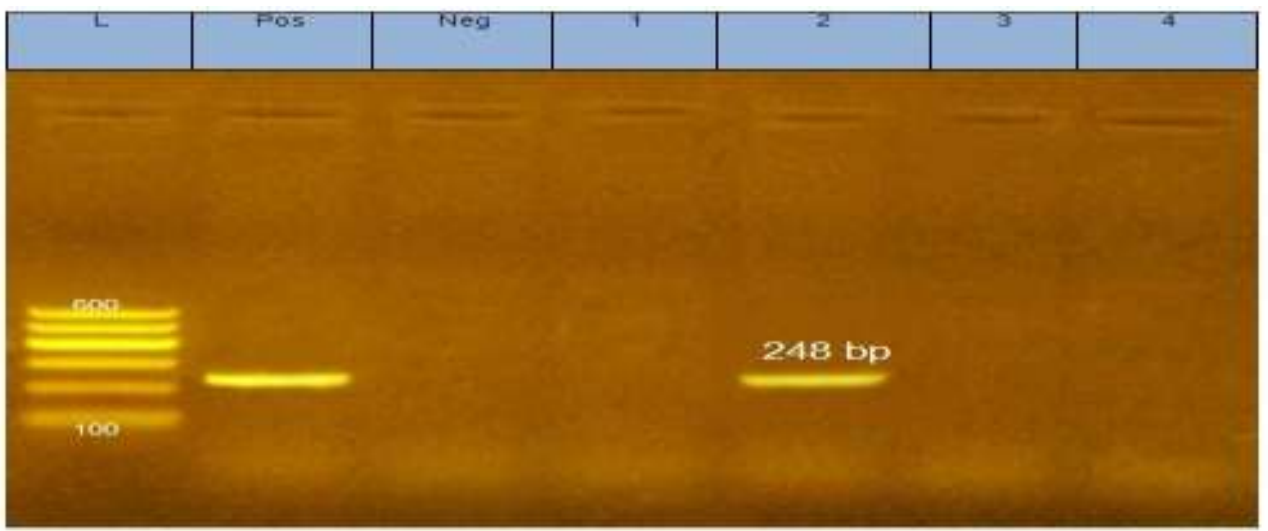

Fig 3:-Agrose gel electrophoresis of PCR amplified products of virulence gene. Lane L: DNA molecular size marker (100bP), lane Neg: Negative control, lane Pos: Positive control, lane 2: eaeA virulence gene of E.coli. The size in base pairs $(248 \mathrm{bP})$ of PCR product is indicated for the bands.

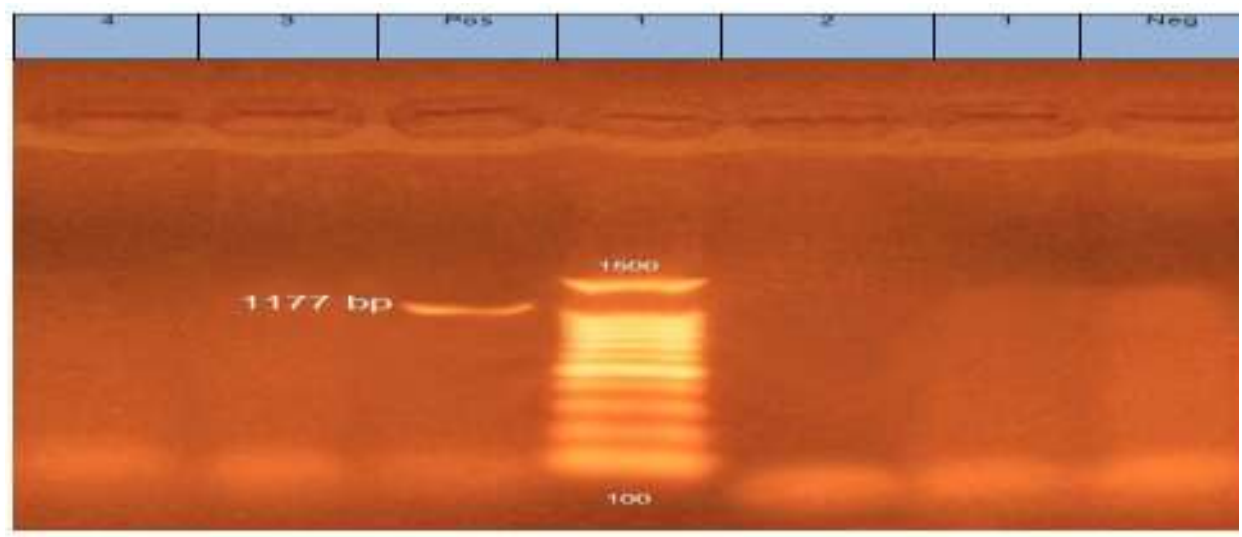

Fig 4:-Agrose gel electrophoresis of PCR amplified products of virulence gene. Lane L: DNA molecular size marker (100bP), lane Neg: Negative control, lane Pos: Positive control of Hly virulence gene of E.coli. The size in base pairs (1177bP) of PCR product is indicated for the bands.

Table 10:- Results of molecular identification of invA and sefA gene of Salmonella.

\begin{tabular}{|l|l|l|l|}
\hline TargetMO & Sample & Results & sefA \\
\cline { 3 - 5 } & & invA & + \\
\hline \multirow{5}{*}{ Salmonella } & 1 & + & + \\
\cline { 2 - 5 } & 2 & + & + \\
\cline { 2 - 4 } & 3 & + & + \\
\cline { 2 - 4 } & 4 & + & + \\
\cline { 2 - 4 } & 5 & + & + \\
\hline
\end{tabular}




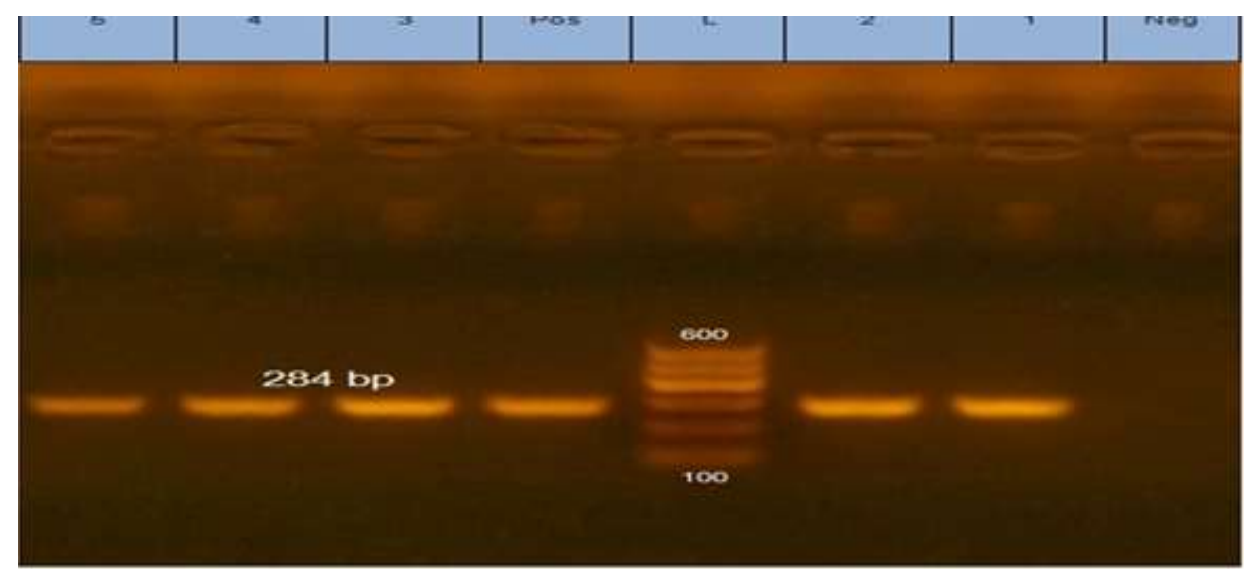

Fig 5:-Agrose gel electrophoresis of PCR amplified products of virulence gene. Lane L: DNA molecular size marker (100bP), lane Neg: Negative control, lane Pos: Positive control, lane 1, 2, 3, 4 and 5: invA virulence gene of Salmonella. The size in base pairs (284bP) of PCR product is indicated for the bands.

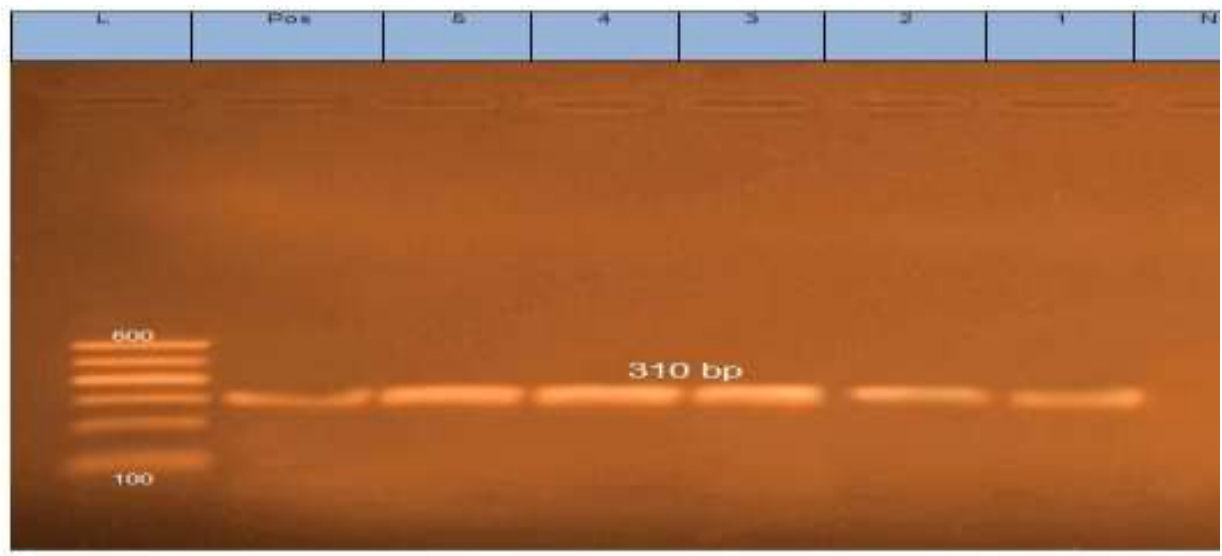

Fig 6:-Agrose gel electrophoresis of PCR amplified products of virulence gene. Lane L: DNA molecular size marker (100bP), lane Neg: Negative control, lane Pos: Positive control, lane 1,2,3,4 and 5: sefA virulence gene of Salmonella. The size in base pairs (310bP) of PCR product is indicated for the bands.

\section{Discussion:-}

Staphylococcus species are potential pathogens, and high population of these bacteria indicates the degree of spoilage which might have undergone(Ali 2014).

In this study, Staphylococcusspp. isolated from Tilapia (Oreochromisniloticus) with an incidence of (19.6\%) as mentioned at (Table 2). While lower incidences of isolation rate recovered by El-olemy et al. (2014), Makilla (2014) and Mus et al. (2014) with an incidences 4.5\%, 0\% and 6\% respectively. But Atwa et al. (2017) isolated $S$. aureus, $S$. epidermidis and $S$. saprophyticus from skin of Tilapia with incidences $12.5,23.8$ and $31.3 \%$ respectively and from liver 15, 12.5 and $16.3 \%$ respectively. While Staphylococcus spp. isolated from Catfish (Claris lazera) with an incidence of (15\%) as mentioned at (Table 3). Our results partial agree with Toyo et al. (2012) reporting incidence $(13.0 \%)$.

On the other hand, Stphylococcusspp. isolated from human with incidences of (24\%) from sellers in markets and (20\%) from workers in farms as mentioned at (Table 4). Higher incidence of Staphylococcus spp. recovered by El-olemy et al. (2014) reporting incidences (35\%) from fish handlers and (37.5\%) from house wife's.

Escherichia coli in fish are considered as an indicator of sewage pollution. E.coli is a bacterium that commonly lives in the intestine of people, animal and fishSoliman et al.(2010 ). 
E.coli isolated from Tilapia (Oreochromisniloticus) with an incidence of (21.4\%) as mentioned at (Table 2). Our results partial similar with Hassan et al. (2012) andSaqr et al. (2016) reporting incidences (27\%) and (18.3\%) respectively. While higher incidences of E.coli were recovered byAmr et al. (2012), David et al. (2009), Galal et al. (2013) and Gupta et al. (2013) reporting incidences 50\%, 57.1\%, 29.34\% and 36\% respectively. But Atwa et al. (2017) isolated E.coli from skin, muscle, intestine and liver with incidences $25,22.5,25$ and $35 \%$ respectively. E.coli isolated from Catfish (Claris lazera) with an incidence of (75\%) as mentioned at (Table 3). Higher incidence of E.coli was recovered by Amr et al. (2012) reporting incidence (100\%). On the other hand, lower incidence of E.coli recovered by Toyo et al. (2015) with an incidence (23.2\%).

On the other hand, E.coli isolated from human with incidences of (20\%) from sellers in markets and (28\%) from workers in farms as mentioned at (Table 4). Our results partially agree with El-olemy et al. (2014) reporting incidences (20\%) from fish handlers and (37.5\%) from house wife's.

Salmonella spp. defined as opportunistic and potential pathogenic bacteria of water bodies in warm climate zones. Salmonella spp. isolated from freshwater fish such as Catfish. This bacterium has a great risk on human health. Budiati et al. (2011)

Salmonella spp. isolated from Tilapia (Oreochromisniloticus) with an incidence of (19\%) as mentioned at (Table 2). Our results agree with Hassan et al. (2012) reporting incidence (21.6\%). Higher incidences of Salmonella were recovered by Nwiyi and Onyeabar (2012) reporting incidences (66.66\%) and (50\%) from whole body and gills respectively. On the other hand, lower incidences of isolation rate recovered by El-olemy et al. (2014) and Makilla (2014) with incidences $(11.5 \%)$ and $(0 \%)$ respectively. Salmonella spp. isolated from Catfish with an incidence of (60\%) as mentioned at (Table 3). While higher incidence of Salmonella spp. recovered by Budiati et al. (2011) with an incidence (80\%). On the other hand, lower incidences of isolation rate recovered by Amr et al. (2012) and Toyo et al. (2015) with incidences (17.1\%) and (7.3\%).

Salmonella spp. isolated from human with incidences of (16\%) from sellers in markets and (24\%) from workers in farms as mentioned at (Table 4). But El-olemy et al. (2014) isolated it with incidences (35\%) from fish handlers and $(25 \%)$ from house wife's.

Pseudomonas spp. is opportunistic pathogens causing disease when the host exposed to stress Magdy et al. (2014). Pseudomonas spp. isolated from Tilapia (Oreochromis niloticus) with an incidence of (11.4\%) as mentioned at (Table 2). But Atwa et al. (2017) isolated $P$. aeruginosa from skin, muscle, intestine and liver with incidences 22.5, $20,17.5$ and $25 \%$ respectively and $P$. fluorescens from the same organs with incidences $20,18.8,15$ and $23.8 \%$ respectively. On the other hand, Pseudomonas spp. isolated from Catfish (Claris lazera) with an incidence of (15\%) as mentioned at (Table 3) but Toyo et al. (2012) isolated Pseudomonas spp. with an incidence (8.7\%).

On the other hand, Pseudomonas spp. isolated from human with incidences (16\%) from sellers in markets and (8\%) from workers in farms as mentioned at (Table 4). Also Sichewo et al. (2013) and Nabih et al. (2016) isolated it.

Aeromonas is an opportunistic and zoonotic important bacterium, Cause diseases in both warm and cold blooded animals as a result of their virulence and pathogenicity Kamble et al. (2012).

Aeromonas spp. isolated from Tilapia with an incidence (10\%) as mentioned at (Table 2). But higher incidences of Aeromonas was recovered by Escarpulli et al. (2003) reporting incidences of A.salmoncidia and A.bestiarum $(67.5 \%)$ and $(20.9 \%)$ respectively and Yagananth et al. (2009) reporting incidence (46.6\%). Aeromonas spp. isolated from Catfish (Claris lazera) with an incidence (10\%) as mentioned at (Table 3). But higher incidence was recovered by Das et al. (2014) reporting incidence of $A$.sobria (77.8\%).

Aeromonas was isolated with an incidence of (0\%) from human as mentioned at (Table 4). While Haenen et al. (2013) reported that Aeromonas transmitted through open wound.

Edwardseilla tarda is commonly classified as opportunistic pathogen; $E$. tarda is a serious pathogen of fish. This bacterium has a great zoonotic importance Lima et al. (2008). 
Edwardseilla tarda isolated from Tilapia with an incidence (1.1\%) and from Catfish with an incidence (0\%) as mentioned at (Table 2, 3). But Mansoer et al. (2014) isolated it with an incidence (40\%) from Asian catfish.

On the other hand, Edwardseilla isolated from human with an incidence of (0\%) as mentioned at (Table 4). While Haenen et al. (2013) reported that Edwardseilla transmitted through open wound.

Ten E.coli isolates were serotyped (six from Tilapia and four from human). Serotyping revealed that five isolates from Tilapia were belonging to $(\mathrm{O} 153, \mathrm{O} 1, \mathrm{O} 125$ and $\mathrm{O} 78)$ and three isolates from human belonging to $(\mathrm{O} 153, \mathrm{O} 26$ and 078) as mentioned at (Table 5). While Barbosa et al. (2014) by serological identification of 49 E.coli revealed that the most common serogroups were O125, O126 and O158.

Ten Salmonella isolates were serotyped (six from Tilapia and four from human). Serotyping revealed that four isolates from Tilapia belonging to $S$. ingada,$S$. typhimurium, $S$. kentucky and $S$. molade. Two isolates from human belonging to $S$. typhimurium and $S$. enteritidis as mentioned at (Table 6). But David et al. (2009) by serological identification of Salmonella revealed that the isolates belonging to $S$. typhimurium, $S$.typhi and $S$. enteritidis.

In this study as mentioned at (Table 7). E.coliO153 strain isolated from fish and human, E.coli O125 and E.coli O78 isolated from fish and E.coli O26 were resistant to Doxicyclin. E.coli O1 isolates was resistant to Ciprofloxacin, Chloramphenicol, Flumequin and Sulphamethoxazol+trimethoprim. E.coli O78 strain isolated from human were resistant to Ciprofloxacin and Chloramphenicol. Soliman et al. (2010) reported that E.coli isolates were sensitive to Enrofloxacine, Oxanilic acid and Spectinomycine. Erythromycine has least effect. Our results disagree with Samuel et al. (2011) who explained that none of E.coli shows resistance to Norfloxacine, Sulphamethoxazol+ trimethoprim and Chloramphenicol. $S$. kentucky isolates were resistant to Erythromycine and Neomycin, $S$. molade and $S$. enteritidis isolates were resistant to Ciprofloxacin. $S$. kentucky, $S$. molade and $S$. enteritidis were sensitive to Amoxicillin and Sulphamethoxazol+ trimethoprim. S. typhimurium isolates were resistant to Penicillin, Chloramphenicol and Erythromycine. S.typhimurium isolated from human was resistant to Ciprofloxacin. But Nesa et al. (2011) reported that the isolated Salmonella serovars from human were highly sensitive to Ciprofloxacin. While El-Hadi (2014) explained the highest antibiotic resistance of Salmonella was observed to Tetracycline (90.71\%) followed by Amoxicillin (70\%) and Amoxicillin+ Clavulanic acid (45\%).

As mentioned at (Table 8) all isolates of Staphylococcusaureus contain clfA gene but not have Hla gene, examination of $c$ lfA gene giving PCR product of (638) bP size and Hla gene giving PCR product of (704) bP size . The prevalence of clfA gene of Staphylococcus aureus was $100 \%$ and Hla gene was $0 \%$. While Abdul-Kareem and Husain (2015) revealed that the prevalence of hly gene of Staphylococcus aureus in samples was $100 \%$. One isolate of E.coli contain eaeA gene and none of the isolates contain Hly gene. Examination of eaeA gene giving PCR product of (248) bP size as shown in (Table 9). The prevalence of eaeA gene of E.coli was 25\%. And this agrees with Kargar and Hamayoon (2015) at which only one isolates from seven isolates of E.coli O157:H7 contains eaeA gene but not has hlyA gene. But Aljanaby and Alfaham (2017) revealed that the lower prevalence of virulence genes in E.coli were (4\%) of eaeA and stxl. As mentioned at (Table 10) all isolates of salmonella contain invA and sefA genes, examination of invA gene giving PCR product of (284) bP size and sefA gene giving PCR product of (310) bP size. The prevalence of invA and sefA genes of Salmonella was 100\%. This agree with Amalia et al. (2014) at which PCR product for invA gene of Salmonella appear at (284) bP size. Moreover, only one sample contains invA gene of $S$.typhimurium and five samples contain sefA gene of $S$. enteritidis yadav et al. (2017).

\section{Conclusion and Recommendation:-}

We can concluded that the most important bacteria causing severe losses in fish are Staphylococcus spp., E.coli, Salmonella spp., Pseudomonas spp., Aeromonas spp. and Edwardseilla spp., these bacteria can transported to human and cause disease. So we recommended the following items:-

1. Adequate cleaning and sanitization of utensils that the fish preserved on it.

2. Effectively training for workers at farms and sellers at markets in hygiene and safety.

3. Application of strict hygienic measures during handling of fish. 


\section{References:-}

1. Abdul-Kareem, H.S. and Husain, A.S. (2015): Genetic comparative study of Staphylococcusepidermidis isolated from wounds, burns and skin flora. Iraq Journal of science., 56(8):708-712.

2. Akbarmehr, J., Salehi, T.Z. and Brujeni, G.H. (2010): Identification of Salmonella isolated from poultry by MPCR technique and evaluation of their hspgroEL gene diversity based on the PCR-RFLP analysis. African Journal of Microbiology Research., 4(15).

3. Ali, H.H. (2014): Isolation and identification of Staphylococcus bacteria from fish of fresh water and its antibiotics sensitivity in Mosul city. Bas. J. Vet. Res., 1(1).

4. Aljanaby, A.A.J. and Alfaham, Q.M. (2017): Phenotypic and molecular characterization of some virulance factors in multidrug resistance Escherichia coli isolated from different clinical infections in Iraq. American Journal of Biochemistry and Molecular Biology., 7(2):65-78.

5. Amalia, U., Hariyadi, R.D. and Poernomo, A. (2014): Rapid detection of Salmonella in shrimp by Polymerase Chain Reaction. J.Teknol. Dan IndustriPangan., 25(1).

6. Amr, A.A., Hosam, A.A. and Heba, R.M. (2012): Enteropathogen of some fresh water fish. Alex J. Vet. Science., 37(1):49-52.

7. Atwa, E.I. (2017): Bacteriological study of fish samples collected from different markets in some Egyptian Governorates and antimicrobial sensitivity of isolates. Int. J. Curr. Microbiol. App. Sci., 6(5):2765-2776.

8. Awuor, W.S., Miruka, O.D. and Eliud, W.N. (2012): Characterization of Salmonella isolated from Nile Tilapia (Oreochromis niloticus) along Lake Victoria beaches in weastern Kenya. World academy of science engineering and technology (International journal of biology. biomolecular. Agriculture. Food and biotechnological engineering., 5(8).

9. Barbosa, M.M., Pinto, F.D., Ribeiro, L.F., Guriz, C.S., Maluta, P.R., Rigobelo, E.C., Avila, F.A. and Amaral, L.A. (2014): Serology and patterns of antimicrobial susceptibility in Escherichia coli isolates from pay- to-fish ponds. Arq. Int. Biol. Sao Paulo., 81(1):43-48.

10. Bisi-Johnson, M.A., ObiCL, Vasaikar, S.D., Baba, K.A. and Hattori, T. (2011): Molecular basis of virulence in clinical isolates of Escherichia coli and Salmonella species from a tertiary hospital in the Eastern Cape, South Africa. Gut Pathogens., 3:9.

11. Budiati, T., Rusul, G., Alkarkhi, A.F.M., Ahmed, R. and Arip, Y.M. (2011): Prevalence of Salmonella spp. from Catfish (Claris garipinus) by using improvement isolation methods. International conference on Asia agriculture and animal IPCBEE., 13.

12. Bujjama, J. and Padmavathi, P. (2015): Prevalence of Staphylococcusaureus in fish samples of local domesyic fish market. International Journal of Current Microbiology and applied science., 4(5):427-433.

13. Cagatay, I.T. and Sen, E.B. (2014): Detection of pathogenic Aeromonashydrophila from Rainbow trout (Oncorhynchusmykiss) farms in Turkey. International Journal of agriculture and biology., 16(2):435-438.

14. Das, A., Rathore, A., Janen, C., Hemanth, C. and Balakrishnan RA. (2013): Diagnosis of motile Aeromonas sobria from Catfishes with septicemia by PCR. IOSR Journal of agriculture and veterinary science (IOSR- JAVS)., 2(6):8791.

15. David, O.M., Wandili, S., Kakai, R. and Waindi, E.N. (2009): Isolation of Salmonella and Shigella from fish harvested from the winam gulf of lake Victoria, Kenya. J. infect developing countries., 3(2):99-104.

16. Denev, S., Staykov, Y., Moutafchieva, R. and Beev, G. (2009): Microbial ecology of the gastrointestinal tract of fish and the potential application of probiotics and prebiotics in finfish aquaculture. Int. Aquat. Res., 1: 1-29.

17. Eissa, N.M.E., Abou El-Ghiet, E.N., Shaheen, A.A. and Abbass, A. (2010): Characterization of Pseudomonas species isolated from tilapia (Oreochromisniloticus) in Qaroun and Wadi- Elrayan Lakes, Egypt. Global Veterinaria., $5(2): 116-121$.

18. Elhadi, N. (2014): Prevalence and antimicrobial resistance of Salmonella spp. in raw retail frozen imported fresh water fish to eastern province of Saudi Arabia. Asian Pac. J. Trap Biomed., 4(3):234-238.

19. El-Hady, M.A. and Samy, A.A. (2011): Molecular typing of Pseudomonas species isolated from some cultured fishes in Egypt. Global Veterinaria., 7(6):576-580.

20. El-olemy, G.M., Lobna, M.A., Salem, N., Khalifa, O. and Abd el wahab, M.S. (2014): Detection of some bacterial zoonosis in market fish in Qalyoubia province and their control. Benha Veterinary Medical Journal., 26(2):126-136.

21. Escarpulli, G.C., Figueras, M.J., Arreola, G.A., Soler, L., Rendon, E.F., Aparicio, G.O., Guarra, j. and Chacon, M.R. (2003): Characterization of Aeromonas spp. isolated from frozen fish intended for human consumption in Mexico. International Journal of food Microbiology., 84:41-49.

22. Fei, W., Hongjun, Y., Hong-bin, H., Changfa, W., Yundong, G., Qifeng, Z., Wiaohong, W. and Yanjun, Z. (2011): Study on the hemolysin phenotype and the genetype distribution of staphylococcusaureus caused bovine mastitis in Shandong dairy farms. Intern. J. Appl. Res. Vet. Med., 9(4).

23. Galal, H.M., Hakim, A.S. and Dorgham, S.M. (2013): Phenotypic and virulence genes screening of Escherichia coli strains isolated from different sources in delta Egypt, Life science journal., 10(2).

24. Gupta, B., Ghatak, S. and Gill, J.P.S. (2013): Incidence and virulence properities of E.coli isolated from fresh fish and ready-to-eat fish products. Vet world., 5-9. 
25. Haenen, O.I.M., Evans, J.J. and Berthe, F. (2013): Bacterial infections from aquatic species, potential for and prevention of contact zoonoses. Rev. Sci. Tech, Off. Int. Epiz., 32(2):497-507.

26. Hassan, A.H.M., Noor Eldeen, A.E., Galal, H.M., SohadDorgham, M., Bakry, M.A. and Hakim, A.S. (2012):Further characterization of Enterobacteriaceae isolated from cultured fresh water fish in KafrElshiekh governorate, Clinical, Biochemical and Histopathological study with emphasis on treatment trials. Global Veterinaria., 9(5):617-629.

27. Kamble, S.R., Meshram, U. and Shanware, A.S. (2012): Characterization of Aeromonas species isolated from diseased fish using ERIC-RAPD markers. Aspav. J. MOL. Biol. Biotechnol., 20(3):99-106.

28. Kargar, M. and Hamayoon, M. (2015): Prevalence of shiga toxins (stx1, stx2), eaeA and hly genes of Escherichia coli O157:H7 stains among children with acute gastroenteritis in southern of Iran. Asian Pacific Journal of tropical medicine., 24-28.

29. Kauffman, G. (1974): Kauffmann white scheme. J. Acta. Path. Microbiol. Sci., 61: 385.

30. Kok, T., Worswich, D. and Gowans, E. (1996): Some serological techniques for microbial and viral infections. In Practical Medical Microbiology (Collee, J.; Fraser, A.; Marmion, B. and Simmons, A., eds.), 14th ed., Edinburgh, Churchill Livingstone, UK.

31. Koneman, E.W., Allen, S.D., Dowell, V.R. and Summers, H.W. (1983): Color atlas and text book of diagnostic microbiology. 2nd Ed. J.B. Lippincott, New York, London.

32. Lima, I.C., Fernandes, A.A., Costa, A.A.P., Velasco, F.o., Leite, R.c. and Hackett, J.L. (2008): Isolation and characterization of Edwardseillatarda from Pacumyleusmicans. Arq. Bras. Med.Vet. Zootec., 60(1):275-277.

33. Magdy, I.H., Elhadi, M.A., Ahmed, H.A., Elmeadawy, S.A. and Kenwy, A.M. (2014): Acontribution on Pseudomonasaeruginosa infection in African Catfish (Claris gariepinus). Research Journal of Pharmaceutical, Biological sciences., 5(5).

34. Makilla, D.M. (2014): Fish quality survey: Staphylococcusaureus and Salmonella spp. informal markets of Nairobi. Food science and quality management., 33.

35. Mansoer, A.J., Mahdi, S.S. and Raesan, S.J. (2014): Isolation and identification of Edwardseilla tarda from the middle intestine of the (Asian Catfish) Silmrustriostegus (Heckel, 1843). Mesopot. J. Mar. Sci., 29(1):1-4.

36. Mason, W.J., Blevins, J.S., Beenken, K., Wibowo, N., Ojha, N. and Smeltzer, M.S. (2001): Multiplex PCR Protocol for the Diagnosis of Staphylococcal Infection. Journal of Clinical Microbiology., 39(9).

37. Mus, T.E., Cetinkaya, F. and Umutcelik. (2014): Occurence of Vibrio, Salmonella and Staphylococcusaureus in retail fresh fish, Mussel and shrimp. Actavet. Brona., 83:75-78.

38. Nabih, M.M., Zaki, V.H. and El-Gohary, A.H. (2016): Assesment of some bacterial zoonotic Microganisms from market fishes in four Nile Delta fish species. Asian journal of agriculture research., 1819-1894.

39. Nesa, M.K., Khan, M.S.R. and Alam, M. (2011): Isolation, Identification and Charachterization of Salmonellaserovars from Diarroeic stool samples of human. Bangl. J. vet. Med., 9(1):85-93.

40. Novotny, L., Dvorska, L., Lorencova, A., Beran, V. and Pavlik, L. (2004): Fish: Apotential source of bacterial pathogens for human beings. Vet. Med. Zech., 49(9): 343-358.

41. Nwiyi, P., Onyeabor, A. (2012): Occurance of Salmonella spp. from fresh fish (Tilapia Nilotica Linn) using improved isolation methods.Online Journal of animal and feed research., 2(6):475-478.

42. Olivera, S.D., Rodenbusch, C.R., Ce, M.C., Rocha, S.L.S. and Canal, C.W. (2003): Evaluation of selective and non selective enrichment PCR procedures for Salmonella detection. Lett. Appl. Microbiol., 36: 217-221.

43. Piva, I.C., Pereira, A.L., Ferraz, L.R., Silva, R.S.N., Vieira, A.C., Blanco, J.E., Blanco, M., Blanco, J. and Giugliano, L.G. (2003): Virulence Markers of EnteroaggregativeEscherichia coliisolated from children and adults with diarrhea in Brasília, Brazil. Journal of Clinical Microbiology., pp. 1827-1832.

44. Quinn, P.J., Carter, M.E., Markey, B.K., Donnelly, W.J.C. and Leonard, F.C. (2002): Veterinary Microbiology and Microbial diseases. MPG. Book 1st ed. Bodmin, Cornwall, UK.

45. Samuel, L., Marian, M.M., Apun, K., Lesley, M.B. and Son, R. (2011): Characterization of Escherichia coli isolated from cultured Catfish by antibiotic resistance and RAPD analysis. International food research journal., 18(3):971-976.

46. Saqr, S., Khalie, R. and Ibrahim, M.S. (2016): Antibiotic resistance and virulence genes of E.coli isolated from fresh Nile tilapia (Oreochromis niloticus) in El- Behera Governorate, Egypt. Alexandria journal of veterinary science., 48(2):83-90.

47. Srivani, R. (2001): Studies on antimicrobial susceptibility pattern of Salmonella isolates from Chennai, India. Inter. J. Pharma and Bio Sciences., 2: 435 - 442.

48. Sichewo, P.R., Gono, R.K., Muzvondiwa, J.U. and Sizanobuhle, N. (2013): Isolation and identification of pathogenic bacteria in edible fish: A case study of Fletcher Dam in Gweru, Zimbabwe. International Journal of science and research (IJSR), India online., 2(9).

49. Sing, C.K., Khan, M.Z.I., Daud, H.H.M. and Aziz, A.R. (2016): Prevalence of Salmonella spp. in African Catfish (Claris gariepinus) obtained from farms and wet markets in Kelantan, Malaysia and their antibiotic resistance. SainsMabysiana., 45(11):159-1602.

50. Sambrook, J., Fritscgh, E.F. and Mentiates. (1989): Molecular coloning. A laboratory manual. Vol!., Cold spring Harbor Laboratory press, New York. 
51. Soliman, M.k., Khalil, R.H., Saad, T.T., El-Gamal, M.H.L. and Gebrail, A.E. (2010): Isolation and identification of E.coli from cultured fresh water. Journal of the Arabian aquaculture society., 5(1).

52. Tayo, A.B.C., Odunn, I.N.J.P.N. and Okonko, I.O. (2012): Microbiological and Physiochemical level of fresh catfish(Arius hendelotic) from different markrts in AkwaIbm state, Nigeria, New York Science Journal., 5(4).

53. Yadav, Kumar, S., Ahaduzzaman, M.D., Islam, Shafiqul, M.D., Siddiki. And Amamzonaed. (2017): Isolation and identification of Salmonella spp. from captive ostriches in Bangladesh by invA gene and sefA gene. Specific PCR and detection of their antimicrobial resistance patterns.Annals of Veterinary and Animal science., 4(1).

54. Yagoub, S.O. (2009): Isolation of Enterobacteriaceae and Pseudomonas spp. From raw fish sold in market in Khartoum state. Journal of Bacteriology research., 1(7): 85-88.

55. Yogananth, N., Bhakyaraj, R., Chanthuru, A., Anbalagan, T. and Nila, M. (2009): Detection of virulence gene in Aeromonashydrophilia isolated from fish samples using PCR technique. Global journal of Biotechnology and Biochemistry., 4(1):51-53. 